Editorial

\title{
Editorial on advanced quantum computers
}

\section{Introduction}

There has been significant advancement in quantum computing; today it's researcher's adventure but one day it will be mainstream. The effects of quantum computing will reach far from research lab. It will be widely used by professionals and developers considering solving problems that once thought unsolvable. Below are few of numerous advanced researches which will aid humanity to move forward. ${ }^{1}$

\section{Construction of quantum computer}

Quantum computers can be built in principle but there are technological challenges. Instead Scientists have designed simple and novel method where voltages are supplied to a quantum computer microchip without having to align many laser beams to the same effect. Professor Winfried Hensinger and his team at Sussex has also succeeded in demonstrating the core building block of new method with an impressively low error rate at quantum computing facility (Figure 1). ${ }^{2,3}$

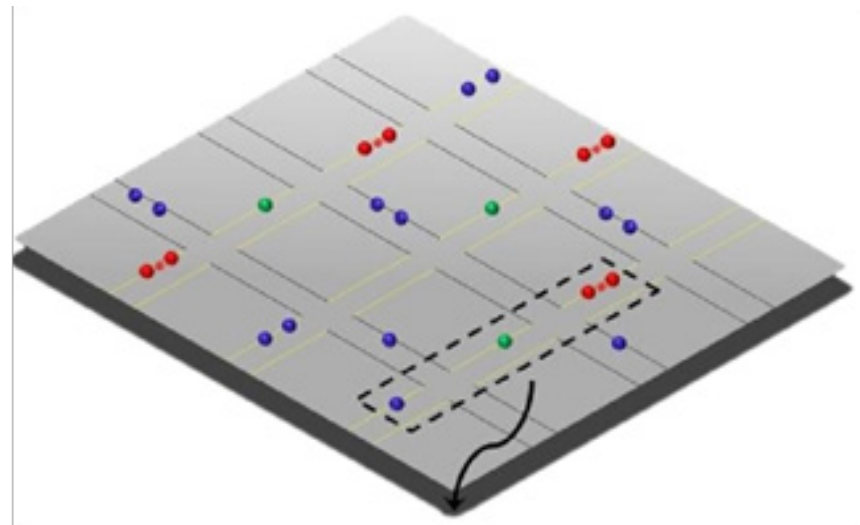

Figure I Illustrates an arbitrary large quantum computer that can be constructed based on simple to engineer approach ${ }^{3}$ image credit: university of sussex.

\section{Connecting quantum internet}

Memories allows to buffer and synchronize quantum information and operations are required for long range quantum communication. Scholars are using Erbium, a rare earth ion that has unique quantum properties that operates in the same band as existing fiber optic networks, eradicating the need for a conversion process. The exceptional advantage of this technology is that it operates in the range of 1550 nanometer band as todays existing networks. They have documented that erbium ions in a crystal can store quantum information for more than a second which is ten thousand times longer than other efforts and is long enough one day to send quantum information throughout global network. ${ }^{4,5}$

\section{Quantum matrices}

Academics have constructed Quantum Matrices using quantum gates. They have processed 65536 quantum bits utilizing 16 quantum

\author{
Volume 4 Issue 5 - 2018
}

\section{Manu Mitra}

Golden Key International Honor Society Member, IEEE Member, USA

Correspondence: Manu Mitra, Author, Golden Key International Honor Society Member, IEEE Member, USA, Email manu.ieee@gmail.com

Received: October 16, 2018 | Published: October 23, 2018

circuits to accomplish square, identity and transposition of matrix with respect to phase, angle, complex values and magnitudes. Quantum Matrices solves system performance with big Random Access Memory (RAM) and high clock rate for Quantum Processing Unit (QCPU). It is also used for calculating positive entries when constructing a larger system in a quantum processor; one can still claim that solution exists and it's positive (Figure $2 \&$ Figure 3$){ }^{6}$

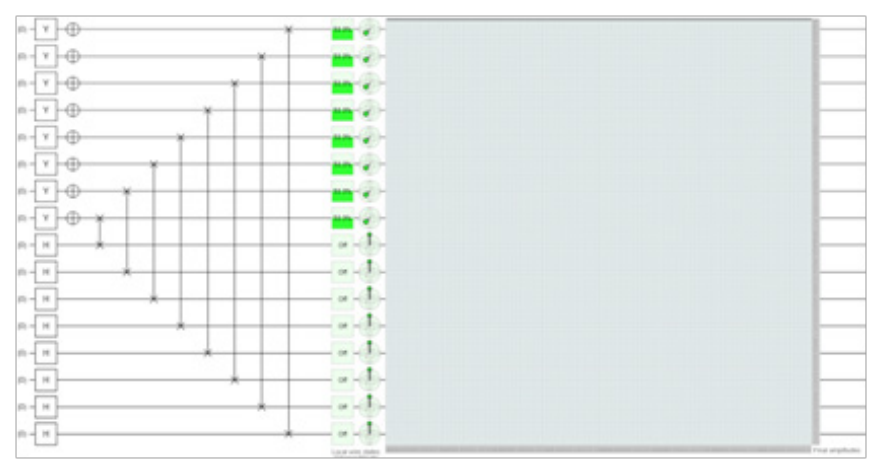

Figure 2 Illustrates before transposition of Matrix using Pauli Y, Hadamard, NOT and swap gates utilizing sixteen quantum circuits and total usage of 256quantum bits. ${ }^{6}$

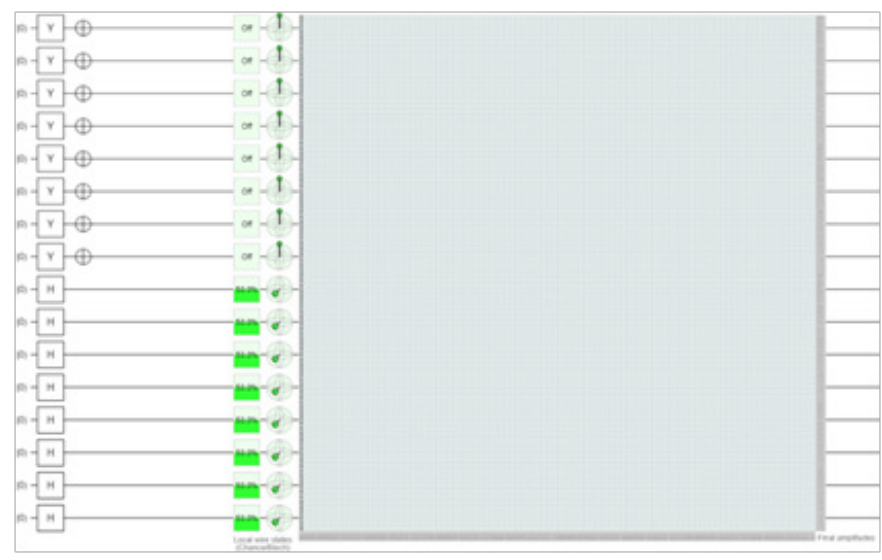

Figure 3 Illustrates after transposition of matrix using Pauli Y, Hadamard, NOT and swap gates utilizing sixteen quantum circuits and total usage of 256 quantum bits. ${ }^{6}$ 


\section{Refrigeration for quantum computers}

Just like ordinary processor, a quantum processor too needs cooling mechanism. In quantum computing, thousands or more logical qubits can be used simultaneously for computation to obtain correct result, every quit has to be reset in beginning of the computation. If qubits are too hot they cannot be initialized because switching between different states too much can result in heating. This problem was solved by Mikko Mottonnen ${ }^{7,8}$ from Aalto University (Figure 4).

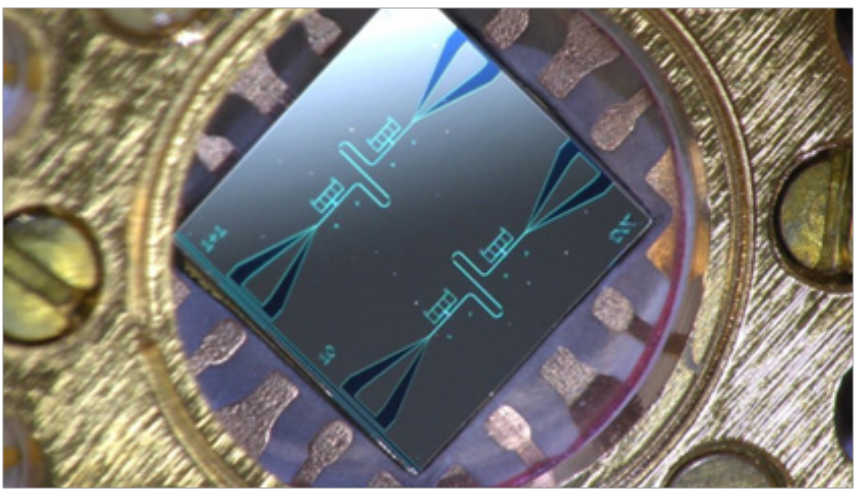

Figure 4 Illustrates centimeter sized silicon chip that has two parallel superconducting oscillators and quantum circuit refrigerators connected to them. ${ }^{8}$

\section{Moving forward with quantum communication}

One of the detriments of quantum communication today is the slow speed of data transfer, which is limited by the speed at which users can perform quantum measurements. Experts at Bar-llan University has invented a new method that overcomes speed limit and enables an increase in the rate of data transfer by more than five order of magnitude. ${ }^{9,10}$ In conclusion, this journal will be committed to continue its consignment to focus with the international research community to achieve the clearest possible scientific picture on the coming up prospect of International Journal of International Robotics \& Automation Journal for betterment of human kind (Figure 5).
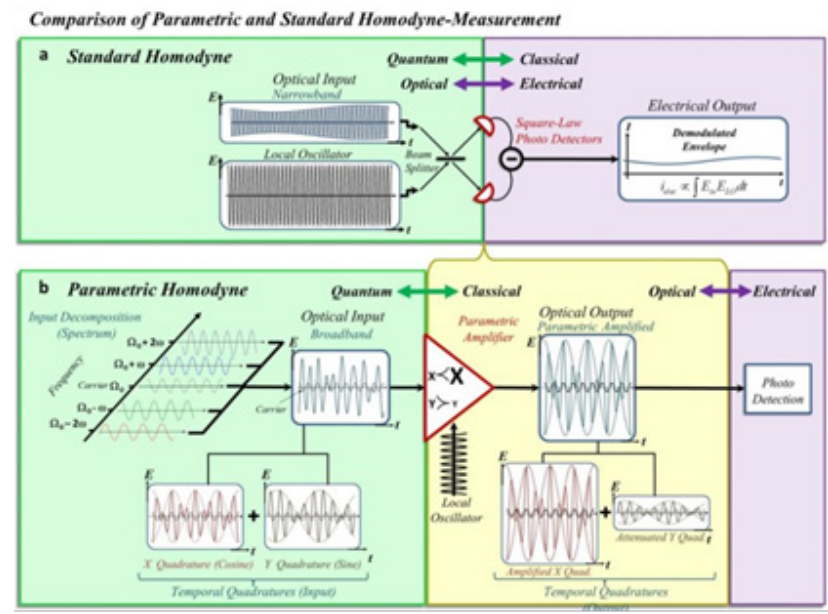

Figure 5 Illustrates quantum communications; participating quantum can detect eavesdropping by resorting fundamental principle of quantum mechanics. $^{10}$

\section{Acknowledgements}

None.

\section{Conflict of interest}

Author declares that there is no conflict of interest.

\section{References}

1. IBM. Quantum computing. IBM Q; USA. 2018.

2. Weidt S, Randall J, Webster SC, et al. Trapped-ion quantum logic with global radiation fields. Physical Review Letters. 2016;117(22).

3. University of Sussex. Construction of practical quantum computers radically simplified: Scientists invent ground-breaking new method that puts quantum computers within reach. Science Daily; 2016.

4. MilošRančić, Morgan P Hedges, Rose L Ahlefeldt, et al. Coherence time of over a second in a telecom-compatible quantum memory storage material. Nature Physics. 2017;14:50-54.

5. Centre for Quantum Computation \& Communication Technology. Connecting up the quantum internet: first practical building blocks for a global quantum internet. Science Daily; 2017.

6. Manu M. Quantum matrices using quantum gates. COJ Elec Communicat. 2018;1(2).

7. Kuan Yen Tan, Matti Partanen, Russell E. Lake, et al. Quantum-circuit refrigerator. Nature Communications. 2017;8:15189.

8. Mikko Mottonnen. Refrigerator for quantum computers discovered. Science Daily; 2017.

9. Yaakov Shaked, Yoad Michael, Rafi Z Vered, et al. Lifting the bandwidth limit of optical homodyne measurement with broadband parametric amplification. Nature Communications. 2018;9(1):609.

10. Forging a quantum leap in quantum communication: Scientists introduce a technique that speeds up quantum information processing nearly a million times. Bar-Ilan University. Science Daily; 2018. 